\title{
Technè
}

La science au service de l'histoire de l'art et de la préservation des biens culturels

41 | 2015

Arts textiles antiques et modernes. Approche scientifique et restauration

\section{Une technique de restauration des textiles appliquée aux peintures modernes et contemporaines : le nettoyage d'un ensemble décoratif de Paul Vera (vers 1924) par compresses d'argile montmorillonite}

A textile restoration technique applied to modern and contemporary paintings : the cleaning of a decorative ensemble by Paul Vera (circa 1924) using Montmorillonite clay compresses

Gilles Barabant, Thalia Bajon-Bouzid et Caroll Fierle

\section{OpenEdition}

Édition électronique

URL : http://journals.openedition.org/techne/4972

DOI : 10.4000/techne.4972

ISSN : 2534-5168

Éditeur

C2RMF

Édition imprimée

Date de publication : 25 novembre 2015

Pagination : 118-126

ISBN : 978-2-7118-6248-1

ISSN : 1254-7867

\section{Référence électronique}

Gilles Barabant, Thalia Bajon-Bouzid et Caroll Fierle, «Une technique de restauration des textiles appliquée aux peintures modernes et contemporaines : le nettoyage d'un ensemble décoratif de Paul Vera (vers 1924) par compresses d'argile montmorillonite », Technè [En ligne], 41 | 2015, mis en ligne le 08 août 2020, consulté le 11 mars 2021. URL : http://journals.openedition.org/techne/4972 ; DOI : https://doi.org/10.4000/techne.4972

\section{cc) (ㅇㅇ $\ominus$}

La revue Technè. La science au service de l'histoire de l'art et de la préservation des biens culturels est mise à disposition selon les termes de la Licence Creative Commons Attribution - Pas d'Utilisation Commerciale - Pas de Modification 4.0 International. 


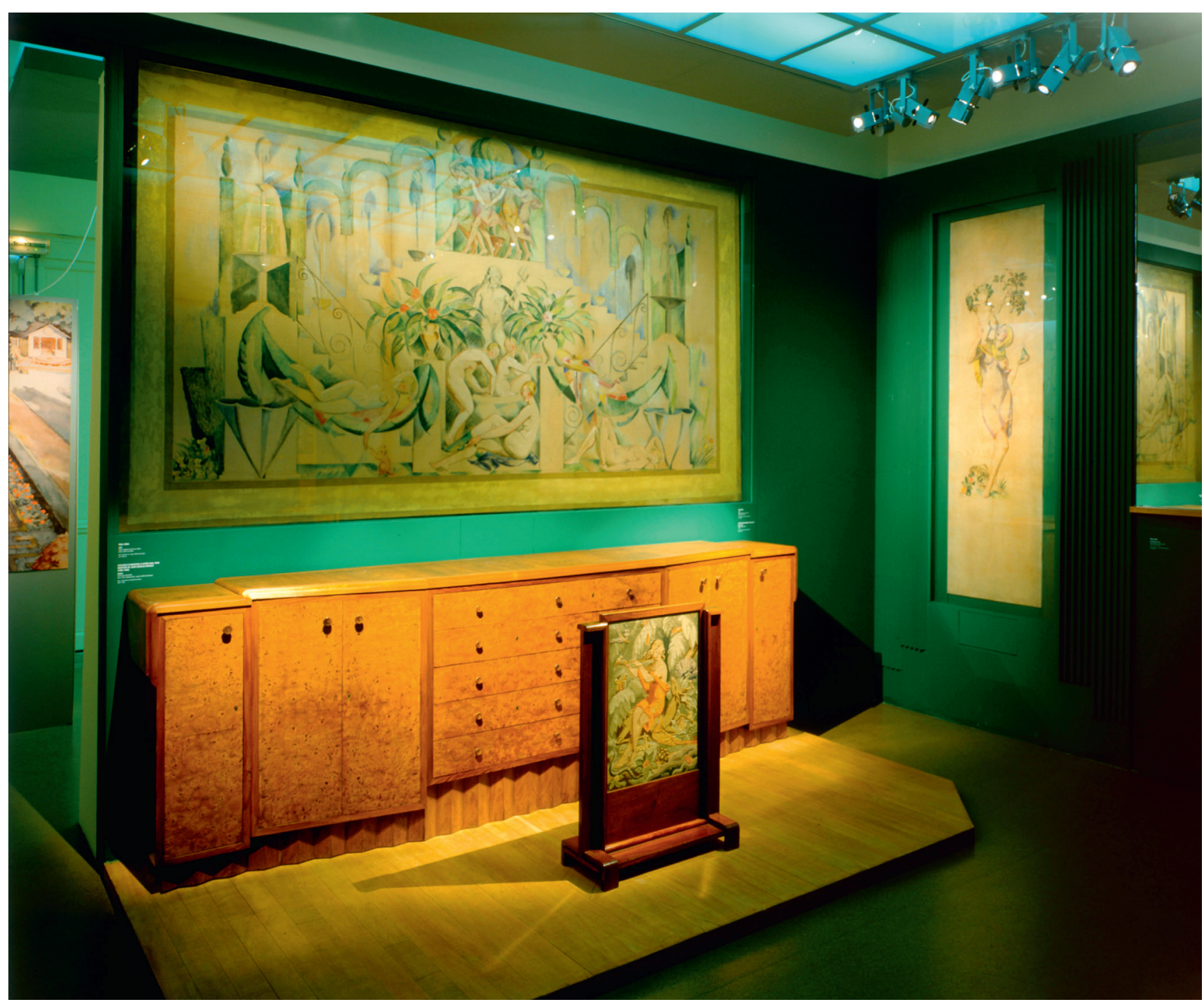

Fig. 1. Vue de la présentation actuelle dans l'espace Paul et André Vera à Saint-Germain-en-Laye. @ Laurent Sully-Jaulmes. 
Gilles Barabant

Thalia Bajon-Bouzid

Caroll Fierle

\section{Une technique de restauration des textiles appliquée aux peintures modernes et contemporaines : le nettoyage d'un ensemble décoratif de Paul Vera (vers 1924) par compresses d'argile montmorillonite}

A textile restoration technique applied to modern and contemporary paintings: the cleaning of a decorative ensemble by Paul Vera (circa 1924) using Montmorillonite clay compresses
Résumé. Le nettoyage d'un ensemble de peintures mates de Paul Vera, gravement défigurées et tachées à cour par des inondations répétées, a pu être réalisé après une phase d'étude préalable associant restaurateur de peinture et restaurateur de textile. L'intervention a été menée en adaptant une technique de compresses d'argile montmorillonite précédemment mise au point et évaluée pour le traitement de tissus. Cette étude de cas montre tout l'intérêt des travaux interdisciplinaires afin de résoudre les problèmes de conservation et de restauration posés par les æuvres modernes et contemporaines et leurs techniques atypiques.

Mots-clés. Peinture mate, auréole, argile, montmorillonite, nettoyage, nettoyage aqueux.
Abstract. A group of Paul Vera's matt paintings, seriously disfigured and stained by repeated leaks, underwent a cleaning process after a preliminary study phase was jointly carried out by a painting conservator and a textile conservator. The operation involved adapting a technique using Montmorillonite clay compresses previously developed and assessed for treating fabrics. This case study demonstrates the advantages of interdisciplinary collaboration in finding solutions for conservation and restoration problems posed by modern and contemporary works and their atypical media.

Keywords. Matt paint, water stain, clay, Montmorillonite, cleaning, aqueous cleaning.

\section{Introduction}

Les peintures du $\mathrm{XX}^{\mathrm{e}}$ siècle, depuis les artistes nabis et fauves, présentent des caractéristiques techniques et esthétiques très différentes des peintures classiques, et des sensibilités tout aussi variables. De ce fait, les méthodes de traitement utilisées pour les œuvres traditionnelles se révèlent souvent inapplicables et doivent faire l'objet d'adaptations, sinon de recherches spécifiques, en vue d'élaborer des solutions alternatives.

Dans la mesure où la plupart des artistes modernes ont privilégié les effets mats dans leurs travaux, la question du nettoyage est sans doute l'une des problématiques les plus fréquentes, les plus complexes et dont la difficulté dépend du degré de matité.

En termes de formulation, une couche picturale mate peut en effet se définir par deux propriétés essentielles que sont l'absence de vernis et l'usage de peintures intrinsèquement mates, c'est-à-dire pauvres en liant. Directement exposées aux facteurs de dégradation, ces œuvres sont donc particulièrement sensibles à l'empoussièrement et à l'encrassement et ces altérations sont difficilement réversibles en raison de la porosité des peintures et des risques mécaniques ou chimiques engendrés par le nettoyage lui-même.
On ne s'étonnera donc pas si, au-delà des problèmes induits par la nature des liants et leur solubilité spécifique, ou des difficultés particulières liées au niveau d'exigence de rendu, le nettoyage des peintures modernes et contemporaines fait l'objet de nombreuses études et recherches appliquées.

La restauration des panneaux décoratifs de Paul Vera appartenant au musée municipal de Saint-Germain-en-Laye (fig. 1) offre ainsi l'exemple convaincant d'un protocole de traitement mis au point pour le nettoyage de textiles, qui a pu être appliqué à des peintures mates considérablement dégradées par des inondations successives.

\section{Le décor de La Thébaïde de Paul et André Vera : heurs et malheurs d'une maison d'artiste}

Les deux frères André (1881-1971) et Paul (1882-1957) Vera ont été d'importants représentants du courant Art déco, qui se sont respectivement illustrés dans l'art des jardins et la peinture décorative ${ }^{1}$. Ils participèrent tout deux, avec l'architecte d'intérieur Jean-Charles Moreux, à l'aménagement de la maison La Thébaïde à Saint-Germain-en-Laye, acquise par André en 1920. Conçu et réalisé entre 1924 et 1935, ce projet

Gilles Barabant, C2RMF, responsable de la filière Xx ${ }^{\mathrm{e}}$ siècle-art contemporain (gilles.barabant@culture.gouv.fr). Thalia Bajon-Bouzid, restauratrice de textiles indépendante (thbajonbouzid@gmail.com).Caroll Fierle, restauratrice de peintures indépendante (c.fierle@wanadoo.fr). 
à valeur de manifeste présentait un ensemble cohérent de décor classique réinterprété à la lumière de la modernité et complété par un jardin aménagé conformément aux théories d'André.

Au décès d'André en 1971, la propriété a été presque immédiatement vendue et détruite. Une part significative du décor et du mobilier a néanmoins pu être sauvée grâce à la donation faite par l'artiste au musée municipal de SaintGermain quelques mois avant sa mort ${ }^{2}$.

Dans le cadre de l'aménagement de l'espace Paul et André Vera initié par Agnès Virole, conservateur du musée, la donation a fait l'objet d'importantes campagnes de conservation et de restauration entre 2005 et 2008 .
La salle à manger est sans doute l'ensemble le plus significatif qui permet de restituer l'esprit du lieu. Il est constitué d'un buffet de Moreux, et de cinq peintures allégoriques de Paul Vera : le grand tableau de l'Été, surmontant le buffet, et quatre peintures de lambris, disposées par paire de part et d'autre de la fenêtre et de la cheminée, dont le thème annonce ou prolonge le jardin adjacent.

Ces quatre panneaux, Le Vin, La Volaille, Les Légumes et Le Lait, avaient tout particulièrement souffert d'inondations répétées et probablement prolongées après leur dépose. L'ampleur des dégradations visuelles engendrées par de spectaculaires auréoles, leur imprégnation dans des aplats de couleur mats posaient la question préalable de la faisabilité même d'une restauration (fig. 2).
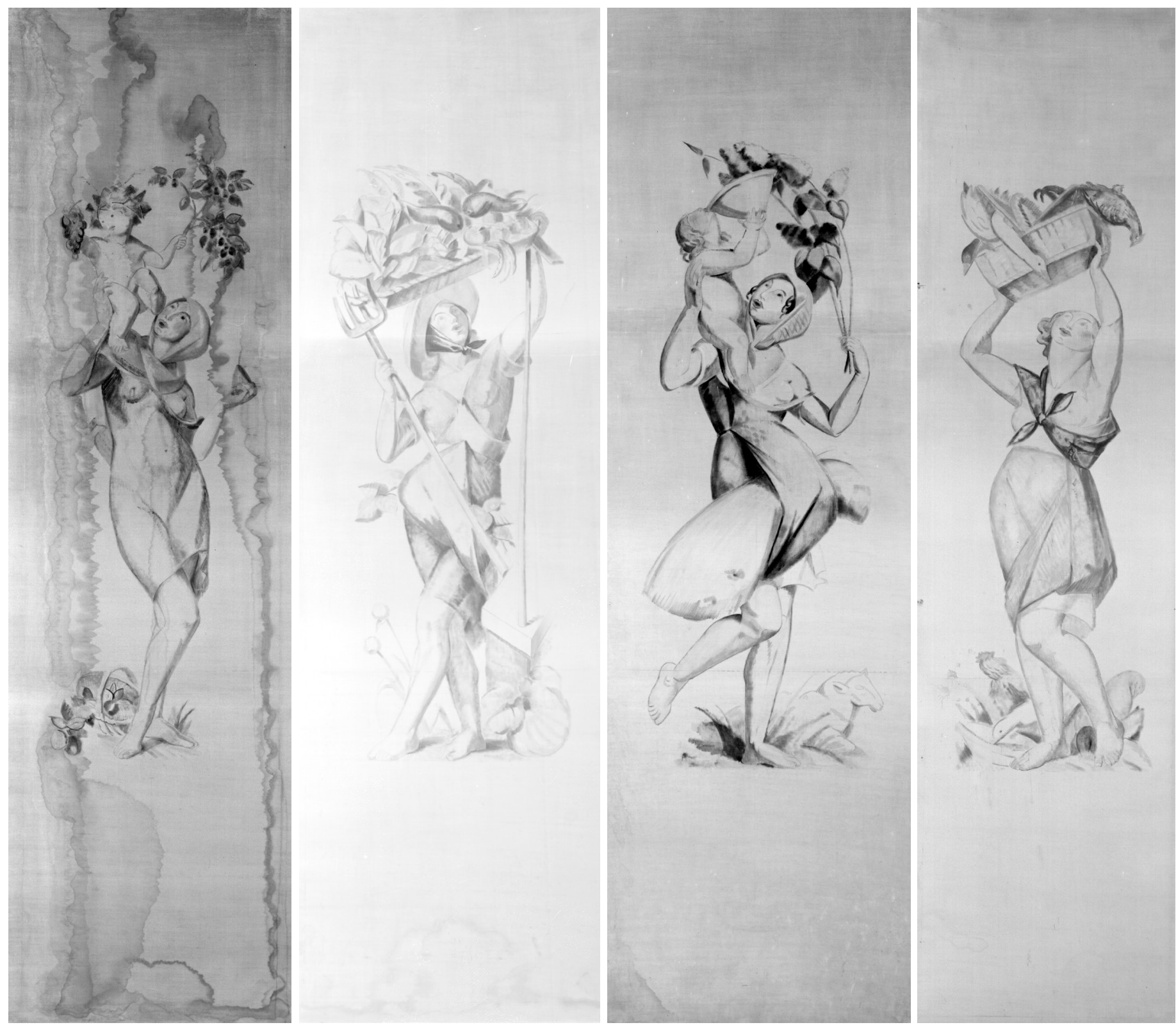

Fig. 2. État des quatre panneaux, avant restauration (noir et blanc) @ C2RMF/Gérard de Puniet et Marc de Drée. 
Afin de lever l'hypothèque, les œuvres ont donc été transportées au C2RMF pour y réaliser un protocole de tests visant à évaluer la compatibilité et l'efficacité des différentes méthodes de nettoyage.

\section{Les panneaux : analyse technique et dégradations}

Les compositions de grand format ${ }^{3}$ sont très homogènes, tant par leur technique que par leur aspect, et nous sont parvenues sur leurs châssis d'origine - châssis fixes, sans chanfrein et de très faible section afin de s'intégrer dans les lambris dessinés par Moreux ${ }^{4}$.

Les toiles de coton utilisées comme support ont une armure toile à tissage serré, et présentent une contexture identique $^{5}$. Elles semblent n'avoir été que très légèrement encollées et la peinture a été directement appliquée, sans couche de préparation intermédiaire ; elles ont par conséquent conservé un caractère hydrophile et absorbant, si bien que la composition transparaît au revers, en image fantôme.
La couche picturale est constituée d'un premier badigeon beige maigre et mat, étendu sur l'ensemble des surfaces, qui forme le fond coloré des compositions. En raison de sa finesse, renforcée par le phénomène d'absorption dans les fibres, il ne masque pas la texture de la toile et confère aux panneaux un aspect général volontairement ambigu qui oscille entre peinture de chevalet et textile teint. Considérant l'époque de réalisation et ces caractéristiques visuelles, la détrempe à la colle ou la peinture à l'huile très diluée apparaissent comme les deux techniques les plus probables, mais la ténuité et la faible teneur en liant de la couche n'ont pas permis de réaliser de micro-prélèvement pour caractérisation. Les tests de nettoyage ont cependant montré que la toile n'était pas sensible aux solvants aqueux, ce qui nous oriente vers l'hypothèse d'une peinture à l'huile.

Les figures allégoriques sont tracées en noir au pinceau sur un dessin préparatoire au crayon, qui reste également visible par endroits. Leur mise en couleur a été réalisée sur le badigeon en touches légères et translucides (fig. 3).

Les dégradations structurelles étaient peu nombreuses malgré la finesse des toiles et la faiblesse des châssis.

Fig. 3. Le Vin, détail, après restauration : technique en frottis de peinture sur la toile recouverte de son badigeon beige. (c) C2RMF/Pierre-Yves Duval.

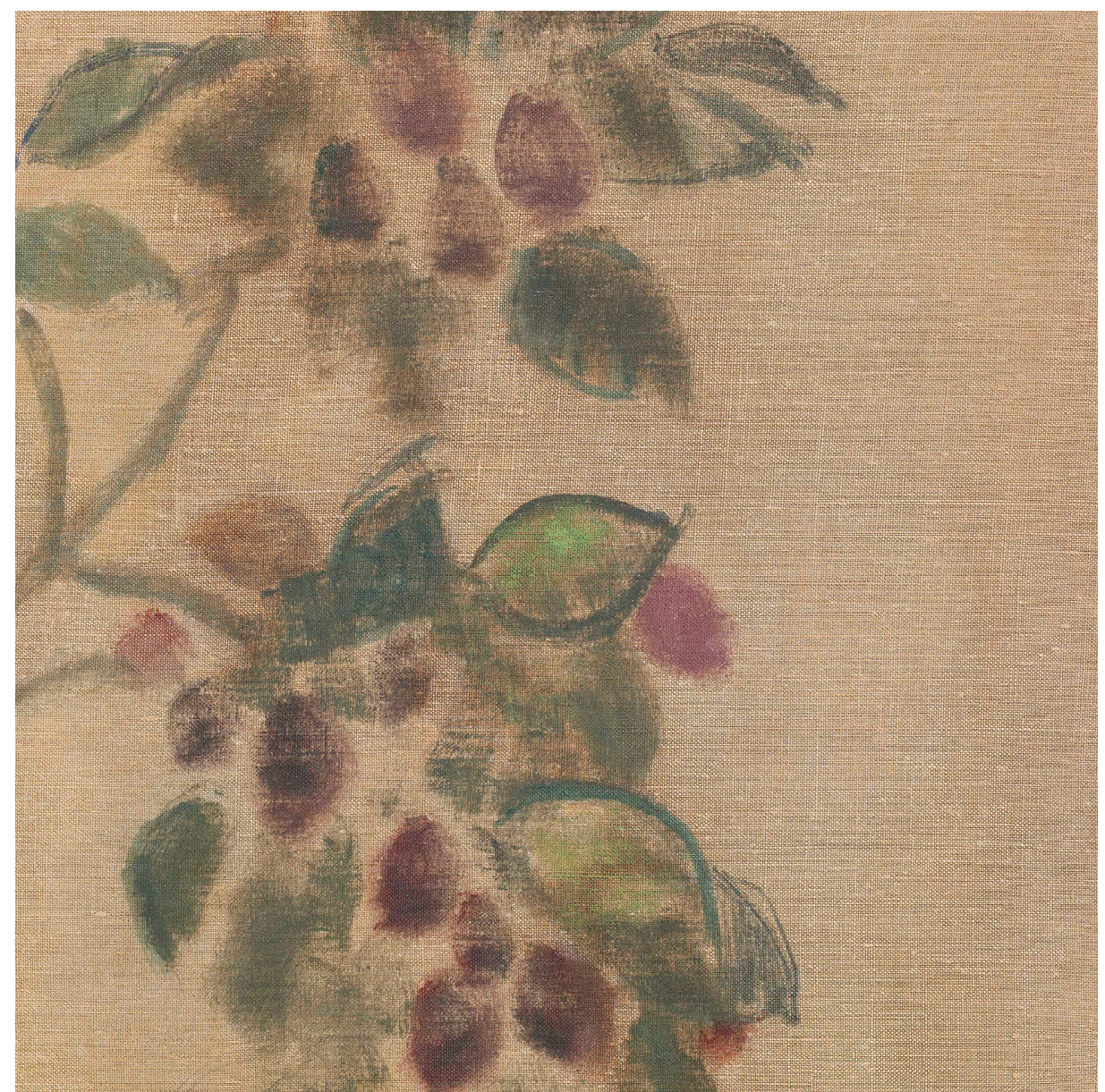




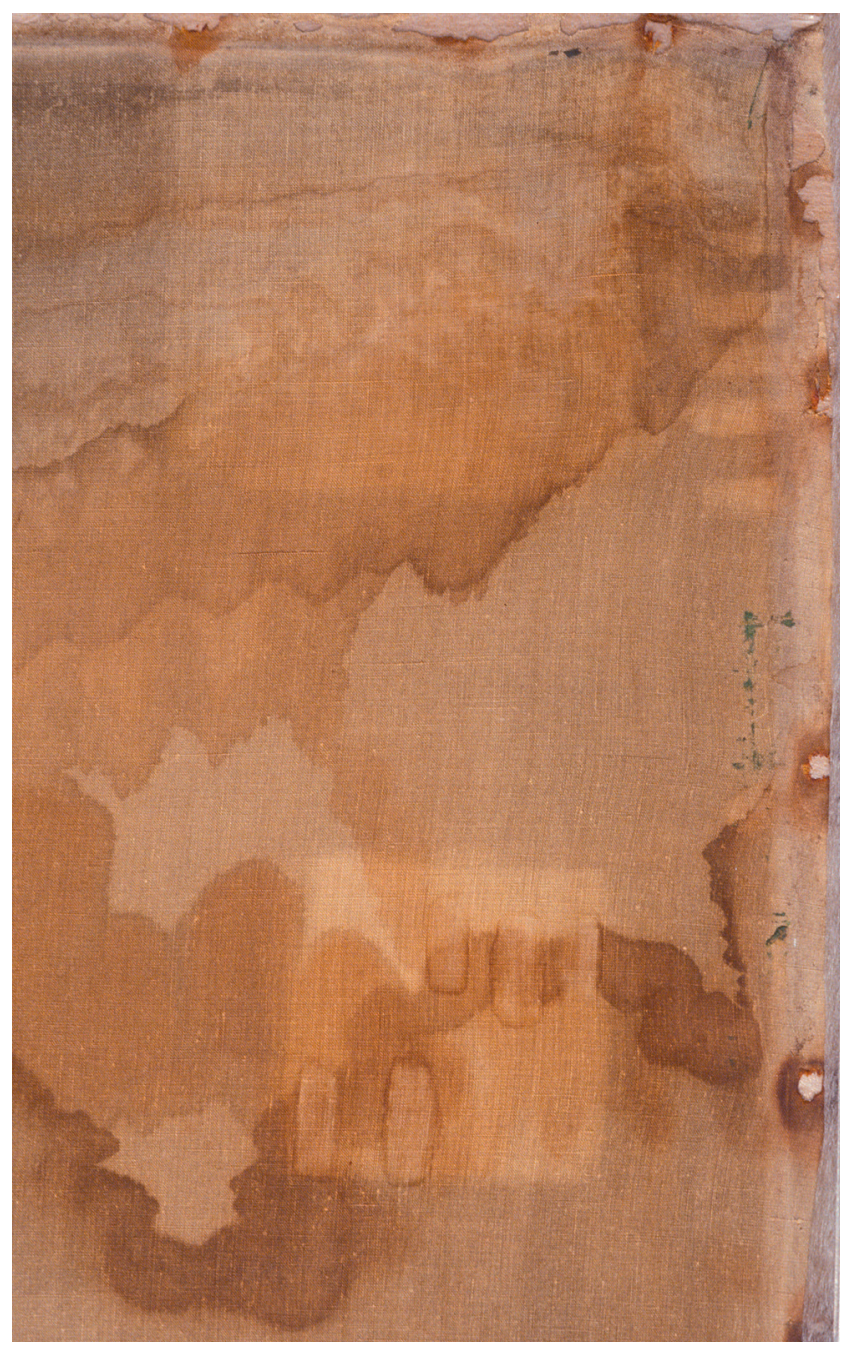

Fig. 4. Le Vin, détail, avant restauration. Auréoles brunâtres et blanchâtres sur le badigeon beige. La fenêtre carrée plus claire, en bas, correspond à un test de gommage, les fenêtres rectangulaires aux tests de nettoyage. (c) C2RMF/Gérard Dufrêne.

L'empoussièrement était en revanche important et général, couvrant les compositions d'un voile gris terne assez homogène et incrusté dans la toile.

Sur Le Lait, La Volaille et Les Légumes, les inondations n'avaient provoqué que des dégâts circonscrits, sous forme de taches assez diffuses généralement cantonnées aux parties basses.

Le Vin, en revanche, présentait des successions d'auréoles brunes et intenses zébrant la peinture sur toute sa hauteur et qui couvraient les deux tiers environ de la superficie. Ces taches peuvent avoir différentes origines : eaux usées d'inondations, poussières, composés extraits du bois du châssis, voire matériaux de l'encollage et du badigeon solubilisés et lessivés. L'imprégnation était telle que ces auréoles étaient également visibles au revers de la toile. S'y mêlaient enfin, le long du bord dextre, des taches blanc grisâtre (fig. 4) pouvant correspondre à des efflorescences de sels.

Le niveau d'altération du panneau, conjugué à la fragilité et à la sensibilité de sa technique, amenait en conséquence à réserver le diagnostic.

\section{Étude préalable}

\section{Premiers tests}

Les essais ${ }^{6}$ réalisés dans le cadre de l'étude ont visé à déterminer, en tenant compte des propriétés intrinsèques des matériaux en présence, le moyen le plus adapté pour supprimer ou réduire les auréoles les plus sombres, et à évaluer la possibilité de restituer un état de présentation esthétiquement acceptable.

Les différents produits et techniques couramment utilisés sur des œuvres ayant subi des dégradations comparables ont été testés (fig. 5).

Cette première phase a montré que les solvants organiques n'avaient pas d'action notable. Les nettoyages aqueux ${ }^{7}$ permettaient d'atténuer les auréoles de manière sensible sans entraîner le dégorgement des couleurs originales, à condition toutefois de limiter le temps ou le nombre d'applications.

En revanche, les mises en œuvre classiques se sont révélées inadaptées pour un traitement, en raison des risques et des inconvénients qu'elles présentaient :

- L'action mécanique liée au passage d'un outil lustre et dégrade rapidement les fibres.

- Les traitements nécessitant plusieurs rinçages usent le badigeon et tendent à le vider de sa couleur.

- Enfin et surtout, toute action de nettoyage localisé entraîne une partie de la crasse vers la périphérie de la zone traitée en y créant un nouveau front d'auréole, quelles que soient la vitesse et la méthode de séchage utilisée.

Afin de s'affranchir des risques mécaniques, l'étude s'est orientée vers les systèmes de type gel et les compresses argileuses, en s'intéressant plus particulièrement aux méthodes de nettoyage appliquées aux textiles, domaine avec lequel l'œuvre présente de nombreuses affinités. Les compresses

\begin{tabular}{lr}
\hline Solution et gels testés & Dissolution \\
\hline Cétones (méthyléthylcétone, acétone) & Aucune \\
\hline Méthyléthylcétone + eau déminéralisée (50:50) & Aucune \\
\hline \hline Éthanol + eau déminéralisée $(50: 50)$ & Légère \\
\hline Eau déminéralisée & Bonne \\
\hline Eau déminéralisée + tensio-actif + rinçage & Légère \\
\hline Eau déminéralisée + agent complexant + rinçage & Bonne \\
\hline Gel de LaponiteTM à 5\% & Très légère \\
\hline Compresse de terre de sommières à 30\% & Très légère \\
\hline Gel d'agar-agar à 1\% & Très légère \\
\hline Borohydrure de sodium $0,5 \%$ ou 1\% (dans eau ou éthanol) & Très légère \\
\hline
\end{tabular}

Fig. 5. Tableau des premiers tests de nettoyage. @ Caroll Fierle. 
aqueuses offrent une grande souplesse d'utilisation et peuvent être adaptées à la sensibilité de l'objet puisqu'il est possible de jouer sur différents paramètres tels que le temps d'application, la concentration initiale en eau ou la porosité du film placé entre compresse et surface à traiter. Elles offrent enfin une garantie de traitement homogène sur des zones prédéfinies, caractéristiques particulièrement intéressantes dans le cas de peintures en aplats circonscrits.

\section{Le nettoyage des textiles par compresses d'argile de type montmorillonite}

Cette technique d'humidification contrôlée a été mise au point, à partir de travaux préexistants, pour nettoyer des textiles archéologiques mais aussi pour les décoller de montages anciens ${ }^{8}$.

La montmorillonite ${ }^{9}$ est une argile à feuillets de couleur blanche dotée d'un pouvoir d'absorption d'eau exceptionnel ; elle possède aussi une grande capacité de réabsorption de l'eau et des impuretés, particulièrement utile pour des traitements de nettoyage qui ne nécessitent ni manipulations, ni renouvellement des compresses en cours de traitement.

Elle a en outre l'avantage d'être plastique à certaines teneurs en eau, ce qui permet d'épouser les aspérités ou les irrégularités éventuelles du support à traiter.

\section{Tests préalables : adaptation du traitement et évaluation des résultats}

Une première série de tests a été effectuée sur le badigeon beige afin d'évaluer l'innocuité du traitement puis de varier les paramètres d'application pour déterminer le protocole le plus adapté. Les trois critères étudiés au cours de cette phase ont été la concentration de montmorillonite ${ }^{10}$ dans l'eau, le temps d'application de la compresse et la nature du film intermédiaire à utiliser.

Les compresses, quelles que soient les modalités d'application, ne provoquent pas de dégradation structurelle sur le badigeon, ni de réaction de retrait brutale de la toile. En revanche, le renouvellement du traitement sur une même zone entraîne un affadissement visible de la couleur d'origine.

Les meilleurs résultats ont été obtenus avec des temps d'application longs, de l'ordre de deux à trois heures, et en choisissant des pâtes assez concentrées en argile afin d'humidifier le plus progressivement possible la toile (40 à 50 grammes de montmorillonite dans $100 \mathrm{ml}$ d'eau déminéralisée), ces conditions permettant de solubiliser et d'absorber la plus grande quantité de crasses dans la compresse.

Le choix du matériau placé à l'interface argile/toile a également une influence sur la qualité et l'homogénéité du résultat. Les membranes cellulosiques microporeuses ${ }^{11}$, utilisées dans le protocole initial de nettoyage des textiles, se sont révélées moins adaptées que le papier japon ${ }^{12}$; ce dernier permet d'obtenir un meilleur fondu et une égalisation en valeur des fronts d'auréoles. Afin d'éviter les risques de dépôt d'argile sur la toile, le papier employé doit être suffisamment épais.

Des essais complémentaires ont été réalisés sur les tracés au crayon et sur les figures peintes pour s'assurer de la compatibilité du traitement sur toutes les parties de l'œuvre.

Comme pour tous les autres tests cependant, un nouveau front d'auréoles apparaît à l'issue du traitement, même s'il peut être légèrement fondu et atténué en fonction des modalités d'application de l'argile et des conditions de séchage.

Il est donc apparu que le nettoyage par compresses de montmorillonite permettait d'atténuer significativement les colorations brunes sans parvenir toutefois à les supprimer. Ce résultat partiel peut s'expliquer par la multiplicité des matériaux présents dans les fibres et leurs sensibilités variables au traitement aqueux.

Par ailleurs, le traitement d'humidification devait être global afin de ne pas créer de nouvelles auréoles ; enfin, il ne serait pas possible de ré-intervenir, sous peine de désaturer de manière irréversible la couleur du badigeon.

Des essais complémentaires de retouches colorées ont été effectués sur le badigeon, dans les zones de tests préalables, afin d'estomper les irrégularités qui demeuraient. Les méthodes étaient là encore restreintes puisque les techniques dites « à sec » étaient les seules possibles. L'emploi de crayons de couleur, avec des mines de dureté moyenne, a montré qu'il était possible d'égaliser le badigeon de manière acceptable et en accord avec la tonalité générale des autres panneaux.

\section{Traitement de restauration}

Un décrassage à sec préalable a été réalisé sur l'ensemble des cinq toiles par gommage ${ }^{13}$.

Le Vin a été démonté de son châssis et maintenu en extension sur un bâti de travail ${ }^{14}$ afin de sécuriser l'œuvre pendant la durée de l'intervention.

La montmorillonite sous forme de pâte ${ }^{15}$ a été étendue sur une surface légèrement plus grande que l'œuvre afin d'éviter l'apparition d'auréoles sur les marges de la toile (fig. 6). Sa surface a été aplanie et lissée au rouleau, puis recouverte d'une feuille de papier japon épais ${ }^{16}$.

La toile a été posée face visible sur ce lit d'argile, afin de mieux surveiller l'évolution du traitement tout au long des trois heures d'application.

La toile une fois positionnée, un film de polyester transparent a été appliqué sur le dispositif pour limiter l'évaporation et éviter un séchage trop rapide de l'argile. Afin de garantir un contact parfait entre la toile et l'argile, l'ensemble a été légèrement aplani au rouleau.

Après une vingtaine de minutes, la surface de l'œuvre était entièrement humidifiée, autorisant la migration progressive des crasses vers l'argile (fig. 7). 

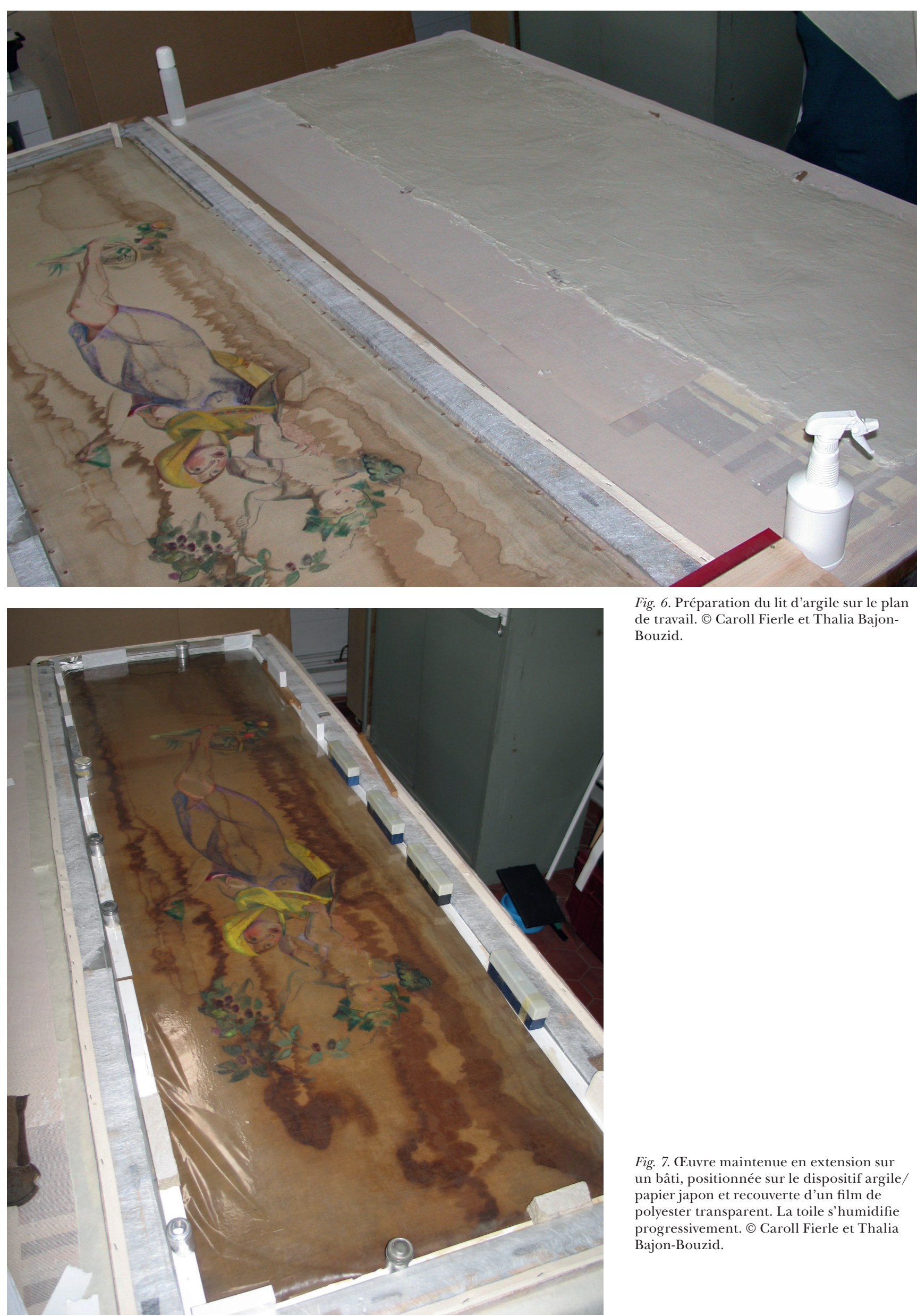

Fig. 6. Préparation du lit d'argile sur le plan de travail. ( ) Caroll Fierle et Thalia BajonBouzid.

Fig. 7. Cuvre maintenue en extension sur un bâti, positionnée sur le dispositif argile/ papier japon et recouverte d'un film de polyester transparent. La toile s'humidifie progressivement. (C) Caroll Fierle et Thalia Bajon-Bouzid. 


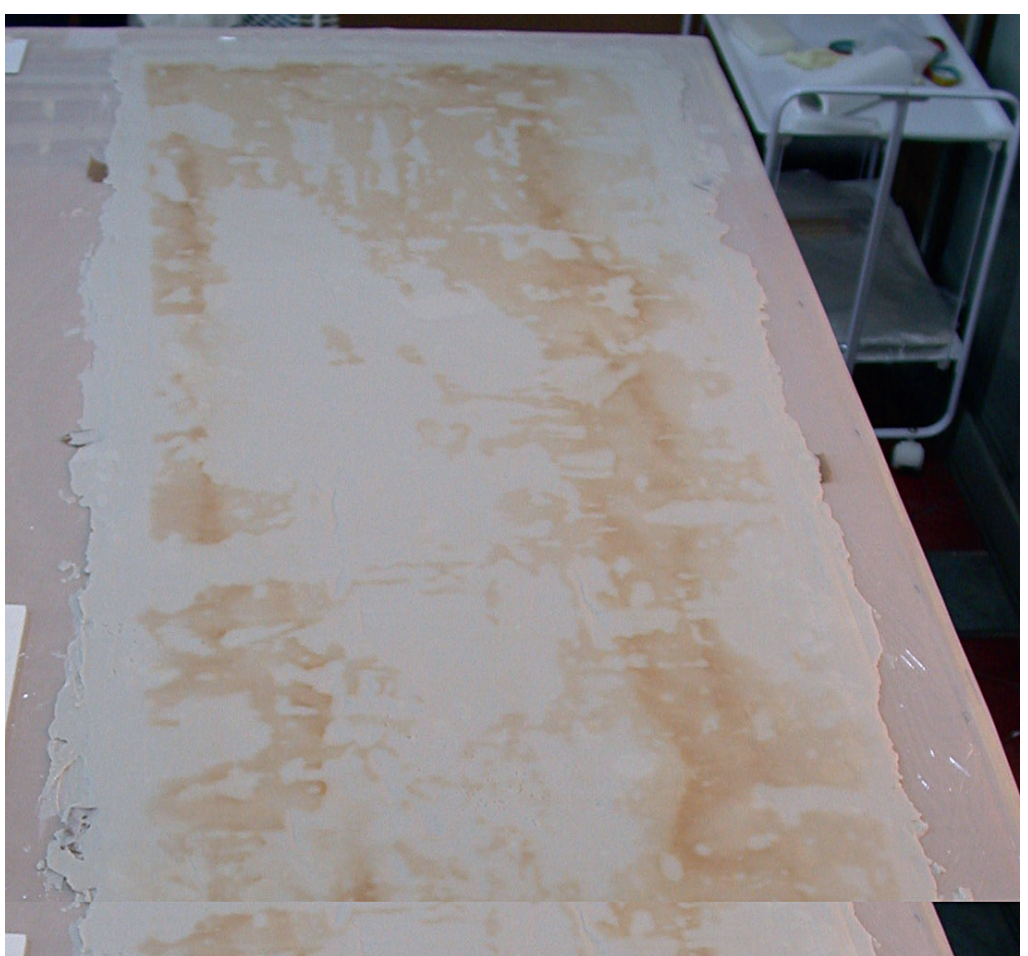

Fig. 8. Couche d'argile après traitement montrant l'absorption des crasses par la montmorillonite. (c) Caroll Fierle et Thalia Bajon-Bouzid.

À la fin du traitement, le film de polyester une fois retiré, la surface entière a été recouverte de buvards non acides afin d'absorber un maximum d'impuretés restées en surface.

À l'examen, ces buvards présentent une coloration brune en décalque parfaite des lignes d'auréoles observées sur l'œuvre, ce qui témoigne de l'efficience du nettoyage.

Après retrait des buvards, le séchage final a progressivement été réalisé à l'aide de sèche-cheveux et de ventilateurs tout d'abord en laissant la toile sur le lit d'argile, puis en soulevant le bâti sur des cales afin d'éviter une nouvelle migration des salissures vers la toile.

Conformément aux conclusions de la phase d'essais, le traitement, s'il a permis d'extraire une part significative des produits d'encrassement (fig. 8), n'a pas fait disparaître les lignes les plus sombres. Il a cependant redonné à l'œuvre une tonalité générale proche de celles des trois autres panneaux et a suffisamment éclairci les auréoles pour la réalisation des retouches colorées.

Afin d'harmoniser Le Vin avec les autres panneaux moins endommagés, cette réintégration ${ }^{17}$ a été menée sur les parties encore tachées (brunes et grises) et sur les zones affadies par la migration de la teinte bistre. Une retouche des éraflures de la couche picturale a également été effectuée (fig. 9).

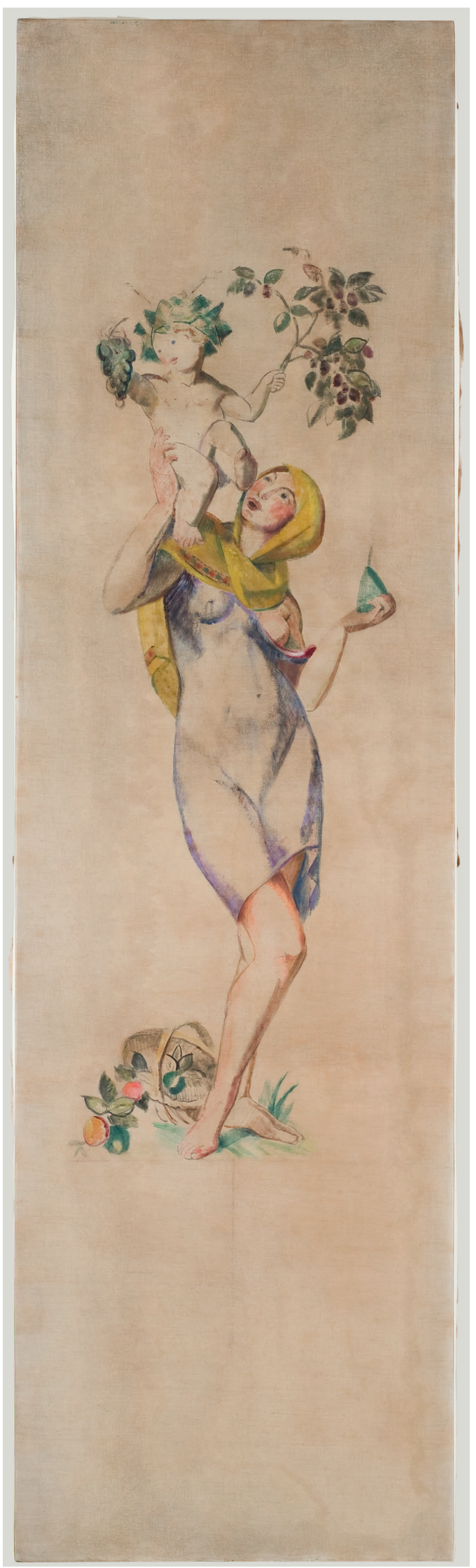

Fig. 9. Le Vin, vue générale, après nettoyage et retouche. (c) C2RMF/Pierre-Yves Duval. 
Dans son état de présentation ainsi restauré, l'œuvre supporte la confrontation avec les trois autres panneaux et a pu être intégrée à la reconstitution de la salle à manger dans l'espace permanent Paul et André Vera à Saint-Germain-enLaye (fig. 1).

\section{Conclusion}

La montmorillonite n'avait pas encore été employée en aussi grande quantité et sur une surface aussi importante. Le caractère expérimental du traitement comme les risques pris lors de l'humification générale se justifiaient cependant par un niveau de dégradation qui n'offrait pas d'alternative.

Mis au point et réalisé dans le cadre d'une démarche interdisciplinaire associant restaurateur de textile et restaurateur de peinture, le protocole d'intervention a montré sa pertinence pour nettoyer des peintures «non classiques » par leurs techniques, leur esthétique ou leurs sensibilités. Il ouvre des perspectives particulièrement prometteuses dans le domaine de la restauration des peintures contemporaines où les problématiques sont complexes, les difficultés souvent cumulatives, et les réponses encore limitées, comme l'illustre le cas des peintures mates, des peintures à liants synthétiques en dispersion ou des peintures monochromes.

La méthode a d'ailleurs été utilisée avec succès depuis lors, par exemple pour nettoyer des peintures vinyliques appliquées en aplats blancs et neutres, tachées à cœur par le contact avec des châssis en cèdre ${ }^{18}$.

\section{Notes}

1. Virole A., Darré A., Day S., Possémé E., 2008.

2. Virole A., 2009, p. 30-32.

3. Dimensions des œuvres : Le Vin: $227 \times 63 \mathrm{~cm} ;$ La Volaille: $227 \times 64 \mathrm{~cm}$; Les Légumes : $227 \times 76 \mathrm{~cm}$; Le Lait: $227 \times 76 \mathrm{~cm}$.

4. Ces châssis trop faibles et en contact avec la toile ont dû être remplacés. Cette phase ultérieure de l'intervention a été réalisée par Claire Bergeaud, restauratrice de peinture, spécialiste des supports toile.

5. Chaîne 27-28 fils/cm et trame 30-31 coups $/ \mathrm{cm}$

6. Bajon-Bouzid Th., Fierle C., août 2006 et juin 2007.

7. Utilisation d'une table bassepression, solution aqueuse additionnée d'un complexant (triammonium citrate, Sigma-Aldrich).

8. Bouzid Th., septembre 2002.

9. Phyllosilicate appartenant à la famille des smectites.

10. Smectite purifiée (CAS number 1327-43-1), Optigel ${ }^{\circledR}$ CL (fournisseur : Rockwood Additives Ltd, France).

11. Boyau cellulosique LCC HS, Viskase $^{\circledR}$ (fournisseur : La Bovida, Paris).
12. Grammage du papier utilisé lors des tests préalables : $10 \mathrm{~g} / \mathrm{m}^{2}$.

13. Gomme en latex vulcanisé Wishab ${ }^{\circledR}$ la plus tendre (fournisseur : Stouls Conservation, Champlan).

14. Montage sur un bâti extensible à l'aide de bandes d'intissé polyester collées au Bévafilm ${ }^{\circledR}$ (fournisseur CTS France, Paris).

15. 40 grammes de montmorillonite dans $100 \mathrm{ml}$ d'eau déminéralisée.

16. Kinugawa $100 \%$ kozo, $22 \mathrm{~g} / \mathrm{m}^{2}$ (fournisseur : Stouls Conservation, Champlan).

17. Polychromos ${ }^{\circledR}$ (Faber-Castell) Prismalo $^{\circledR}$ (Caran d'Ache) et pastels (Conté).

18. Fierle C., 2011 et 2012.

\section{Références bibliographiques}

Virole A., Darré A., Day S., Possémé E., 2008 Paul et André Vera : Tradition et modernité, Hazan, Paris.

Virole A., 2009, « Collections Paul et André Vera : nouvelle présentation ", Revue des Musées de France - revue du Louvre, 3, p. $30-32$.

\section{Documents inédits}

Bouzid Th., 2002, Conserver ou retirer les interventions anciennes : étude de sept textiles islamiques médiévaux restaurés ou montés à la fin du XIX ${ }^{e}$ ou au début du XX $\mathrm{X}^{e}$ siècle. Cataplasme d'argile dans une membrane cellulosique : évaluation d'un dispositif expérimental favorisant une humidification progressive. Mémoire de fin d'études-INP, Département des restaurateurs, septembre 2002

Bouzid Th., Fierle C., août 2006 et juin 2007, Premiers tests de nettoyage sur les auréoles, rapports d'intervention (dossier C2RMF n 60823).

Fierle C., Étude préalable et restauration de 23 panneaux, éléments de l'installation Manifeste de Jean-Pierre Raynaud appartenant au CAPC-musée d'art contemporain de Bordeaux. Intervention réalisée par Caroll Fierle en 2011-2012 (dossier C2RMF n ${ }^{\circ} 67562$ ). 